\title{
Generation of a Radially Polarised Beam in a Solid-State Laser Using an Intracavity Spatially Variant Waveplate
}

\author{
T. L. Jefferson-Brain*, Y. Lei, P. G. Kazansky, W. A. Clarkson \\ Optoelectronics Research Centre, University of Southampton, Southampton, Hampshire, SO17 1BJ, UK \\ *T.Jefferson-Brain@soton.ac.uk
}

\begin{abstract}
Radially polarised laser sources are of considerable interest due to their applications in areas such as laser processing, particle manipulation and high-resolution imaging. Previous approaches for generating radially polarised beams have made use of extra-cavity approaches such segmented waveplates [1], along with intra-cavity methods such as grating mirrors [2] or the use of birefringent crystals [3]. An alternative approach is to use a femtosecond laser-written spatially variant waveplate (S-waveplate) to a convert linearly polarised beam to a radially polarised beam. S-waveplates have the attraction that they can produce radially-polarised beams with very high polarisation purity [4], but, in the past, have suffered from very high scattering loss greatly limiting their usefulness. However, recent research has led to the discovery of a new type of ultrafast-laser-written birefringent modification allowing the writing of S-waveplates with better than an order-of-magnitude lower loss [5]. This latest development opens up the possibility of using S-waveplates as polarisation selecting elements in a solidstate laser to allow direct generation of desirable polarisation distributions.

Here we report on the first demonstration of direct generation of a radially-polarised mode in an end-pumped $\mathrm{Nd}: \mathrm{YVO}_{4}$ laser using a ring-shaped pump beam in combination with a low-loss intra-cavity S-waveplate. The laser yielded $1.3 \mathrm{~W}$ of output at $1064 \mathrm{~nm}$ in a radially-polarised beam with very high polarisation purity. The experimental arrangement employed a simple three-mirror cavity design with an anti-reflection coated 1 at.\% doped $\mathrm{Nd}: \mathrm{YVO}_{4}$ gain medium, end-pumped by an $808 \mathrm{~nm}$ fibre-coupled diode laser. The pump delivery fibre was spliced to a multi-mode capillary fibre-taper with a $200 \mu \mathrm{m}$ outer diameter and $105 \mu \mathrm{m}$ inner air hole; when imaged in the near-field the reshaped pump light forms an annular pump spot to yield a ring-shaped inversion distribution spatially-matched to the desired laser mode. The resonator employed a pump input-coupler at a 45 degree angle of incidence, a planar mirror of $>99.9 \%$ reflectivity at the lasing wavelength $(1064 \mathrm{~nm})$, and a concave outputcoupler $(\mathrm{R}=300 \mathrm{~mm})$ with a transmission of $15 \%$ at the lasing wavelength. The S-waveplate, which was written into an anti-reflection (AR) coated fused silica substrate, was positioned close to the output-coupler.

Without the S-Waveplate in the cavity, the $\mathrm{Nd}: \mathrm{YVO}_{4}$ laser operated on the linearly-polarised $\mathrm{LG}_{01}$ doughnut mode (see Fig. 2a) exploiting the strong polarisation dependent gain in $\mathrm{Nd}: \mathrm{YVO}_{4}$ and yielding a maximum output power of $1.48 \mathrm{~W}$. The intensity profile of the mode obtained with the S-waveplate in the cavity is shown in Fig. $2 \mathrm{~b}$. Figures $2 \mathrm{c}$ and $2 \mathrm{~d}$ show the transmission of the resulting radially polarised mode through a linear polariser at two orientations $90^{\circ}$ apart. By measuring the contrast of the peaks of this two-lobed structure to the intensity minima between them and averaging over many polarizer orientations, the average radial polarisation extinction ratio was determined to be $35: 1$. The maximum output power was reduced slightly to $1.3 \mathrm{~W}$, due to a combination of imperfect antireflection coatings on the S-waveplate substrate and a small amount of scattering from the femtosecond written modifications. By comparing laser performance (i.e. threshold and slope efficiency) in the modified and unmodified substrate regions we were able to deduce that the intrinsic single-pass loss for the Swaveplate was $\sim 1 \%$. The combination of high radial polarisation purity combined with the small increase in cavity loss associated with the S-waveplate suggest that there is considerable potential for further power scaling.
\end{abstract}
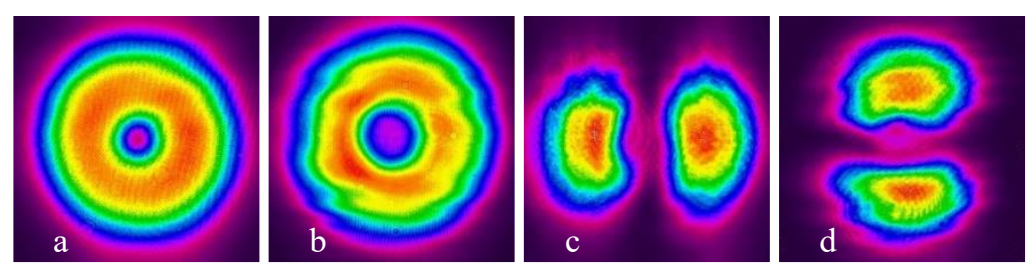

Fig. 1 Images of the transverse intensity profiles obtained from the source. (a) no S-waveplate in the cavity. (b) S-waveplate in the cavity (c\&d) the S-waveplate in the cavity and transmission of the beam through a linear polariser.

\section{References}

[1] G. Machavariani, Y. Lumer, I. Moshe, A. Meir, S. Jackel, Opt. Commun. 281, 732 (2008).

[2] M.A. Ahmed, A. Voss, M.M. Vogel, T. Graf, Opt. Lett. 32, 3272 (2007).

[3] J.W. Kim, J.I. Mackenzie, J.R. Hayes, W.A. Clarkson, Opt. Express 19, 14526 (2011).

[4] Di Lin, J. M. O. Daniel, M. Gecevičius, M. Beresna, P. G. Kazansky, and W. A. Clarkson, Opt. Lett. 39, 5359-5361 (2014).

[5] R. Drevinskas and P. G. Kazansky, "High-performance geometric phase elements in silica glass," APL Photonics 2 (6), 066104 (2017). 